\title{
Effect of the microwave radiation treatment of porous indium phosphide on spectra of radiative recombination centers
}

\author{
R. Red'ko, S. Red'ko \\ V. Lashkaryov Institute of Semiconductor Physics, NAS of Ukraine \\ 41, prospect Nauky, 03028 Kyiv, Ukraine \\ Phone: 38 (044) 525-94-64; e-mail:re_rom@ukr.net
}

\begin{abstract}
To study the influence of microwave irradiation on a spectrum of defect states in porous InP, we have measured the luminescence spectra within the range 0.50 to $2.04 \mathrm{eV}$ at $77 \mathrm{~K}$ before and after short and long (up to $600 \mathrm{~s}$ ) treatments in air in the operation chamber of a magnetron at a frequency of $2.45 \mathrm{GHz}$ and a surface power density of $7.5 \mathrm{~W} / \mathrm{cm}^{2}$. We have obtained that the spectra of defects in researched samples are essentially changed as well as the concentrations of local centers. Possible mechanisms of observable changes in the semiconductor impurity-defect composition caused by a microwave treatment are discussed.
\end{abstract}

Keywords: luminescence, microwave treatment, spectrum of defects.

Manuscript received 08.06.07; accepted for publication 27.09.07; published online 30.11.07.

\section{Introduction}

Indium phosphide is widely used in devices which have been irradiated by microwave sources of different kinds. So the search of effective regimes of treatment with such a radiation to define the extreme regime of exploitation of this material is actual till now. At the same time, the influence of microwave irradiation on the porous InP impurity-defect structure has not been studied yet.

\section{Experimental}

Indium phosphide of the $n$-type with the surface orientation (111) and a concentration of carriers $<<10^{17} \mathrm{~cm}^{-3}$ was the object of our researches. The samples were exposed to microwave irradiation in free space in a centimeter $\left(f=2.45 \mathrm{GHz}, p=7.5 \mathrm{~W} / \mathrm{cm}^{2}\right)$ range of wavelengths. The intensity of the microwave treatment was varied by changing the exposure time.

The defect structure of the samples was studied by using the photoluminescence (PL) method at $77 \mathrm{~K}$ in the spectral range $0.50 \ldots 2.04 \mathrm{eV}$. We used a powerful lamp PZh-100 as a source of exciting radiation. Its emission spectrum was cut with corresponding filters to separate the radiation with $h v \geq 2.0 \mathrm{eV}$. A $\mathrm{PbS}$ photoresistor served as a detector of luminescent light. The temperature of our samples was measured by using a thermocouple (copper-constantan).

\section{Results and discussion}

The dependence of the intensity of PL on the duration of microwave treatment of porous InP, which contained three bands peaking at $0.82,1.02$, and $1.14 \mathrm{eV}$, is shown in the figure.

During the irradiation, the frequency position of $\mathrm{PL}$ peaks does not vary. The influence of microwave radiation on the intensity of PL bands results in the following features.

The intensity of all peaks at small irradiation times $(<10 \mathrm{~s})$ is lowered. But, after a further treatment, it grows until the time of irradiation reaches $200 \mathrm{~s}$. After such a dose of microwave radiation, the intensity of all peaks tends to zero.

As a physical and chemical nature of the centers responsible for the luminescent bands at 1.02 and $1.14 \mathrm{eV}$ is not finally ascertained, we believe in view of [1-3] that they are related to various complexes with participation of the vacancies $\left[\mathrm{V}_{\mathrm{P}}+\mathrm{impurity}\right]$ and $\left[\mathrm{V}_{\mathrm{P}}+\mathrm{Fe}_{\text {In }}\right]$, respectively. There is no information about the band at $0.82 \mathrm{eV}$ in the literature. So, we consider that it is related to the presence of pores on the surface of a sample.

As is well known, a modification of the impuritydefect structure can be caused by the action of the thermal factor. Using a formula proposed in [4] for the estimation a temperature under irradiation due to the presence of impurities 


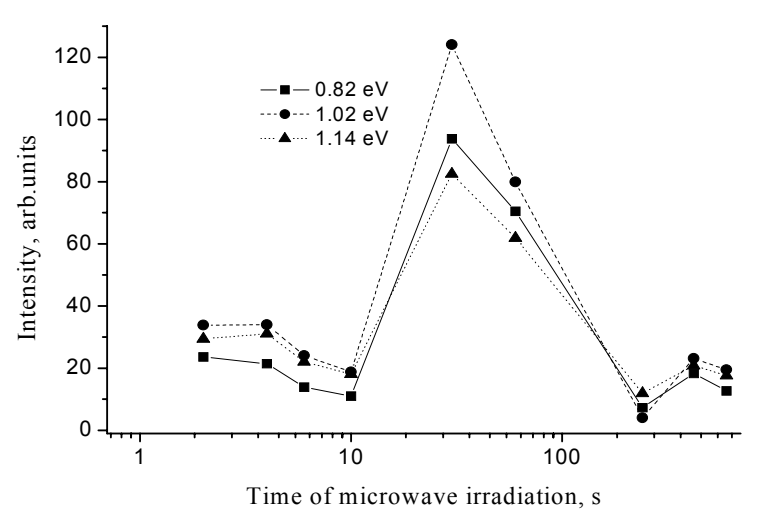

Intensity of the peak of PL bands versus the duration of irradiation.

$$
T_{\max }=T_{0}+\frac{P_{T} R^{2}}{3 k}
$$

where $T_{0}$ is the temperature of the semiconductor-impurity border, $P_{T}-$ density of heat power which enters the impurity area under the microwave energy absorption, and $k$ - factor of heat conductivity, we have obtained that $T_{\max } \approx T_{0}$ (by assuming that $P_{T}$ is the maximum microwave power density). The measurement of the temperature of a sample after the treatment gave $\sim 70^{\circ} \mathrm{C}$. Thus, the temperature factor cannot be a determining one. That is, in order to explain the observed changes, we should look for some nonthermal mechanisms of a modification of the impurity-defect structure of the semiconductor at a small microwave power.

It is possible that a nonmonotonic synchronous change of the intensity of the observed peaks is caused by two rival factors. First of all, there are the nonradiative centers. The synchronous change of the intensity can be caused by changing the concentration of these centers. On the other hand, the microwave treatment can induce the annihilation and generation of initial radiative centers. The destruction of defect complexes is caused, perhaps, by a mechanism related to bonding and antibonding orbitals. According to [5], when an atom absorbs some small energy, its electron can pass from the bonding orbital to an antibonding one. This can result in the destruction of the complex formed by this atom.

\section{Conclusions}

We have observed the nonmonotonic and synchronous change of the intensity of three bands with $h v_{1}=$ $=0.82 \mathrm{eV}, h v_{2}=1.02 \mathrm{eV}$, and $h v_{3}=1.14 \mathrm{eV}$. Analyzing the obtained data, it is possible to draw conclusion that the microwave treatment results in a change of the concentration of local centers which are, probably, both radiative and nonradiative centers of recombination.

Thus, the interaction of a microwave $(f=2.45 \mathrm{GHz}$, $p=7.5 \mathrm{~W} / \mathrm{cm}^{2}$ ) radiation with indium phosphide is accompanied by the action of both thermal and nonthermal factors. It is proposed that, due to the electron transition from the bonding orbital to an antibonding one caused by a microwave treatment, the defect complexes can be destructed.

\section{Acknowledgments}

The authors are grateful to Drs. I.N. Arsent'ev and V.P. Ulin for the provision of samples and to Dr. V.V. Milenin for his permanent interest to our work and the fruitful discussions.

\section{References}

1. A.B. Georgobiani, A.V. Mikulyonok, I.G. Stoyanova et al., Non-equilibrium carrier radiative recombination in indium phosphide single crystals // Phys. status solidi (a) 80, No 1, p. 109-115 (1983).

2. H. Temkin, B.V. Dutt, W.A. Bonner. Photoluminescence study of native defects in InP // Appl. Phys. Lett. 38, No 6, p. 431-433 (1981).

3. H. Temkin, B.V. Dutt, W.A. Bonner et al., Deep radiative levels in InP // J. Appl. Phys. 53, No 11, p. 7526-7533 (1982).

4. A.V. Klyuchnik, Influence of intense microwave radiation on sea ice structure // Zhurnal Tekhnicheskoi Fiziki 62, No 7, p. 99-107 (1992) (in Russian).

5. A.B. Gerasimov, M.S. Gopotishvali, V.V. Dzshibuti, B.M. Konovalenko, On the mechanism of transformation of complexes in semiconductors // Fizika Tekhnika Poluprovodnikov 22, No 5, p. 920-925 (1988) (in Russian). 